\title{
Low-force transitions in single titin molecules reflect a memory of contractile history
}

\author{
Zsolt Mártonfalvi ${ }^{1, \S}$, Pasquale Bianco ${ }^{1, \S}$, Marco Linari ${ }^{2}$, Marco Caremani ${ }^{2}$, Attila Nagy*, Vincenzo Lombardi ${ }^{2}$ \\ and Miklós Kellermayer ${ }^{1,3, \uparrow}$
}

\begin{abstract}
Titin is a giant elastomeric muscle protein that has been suggested to function as a sensor of sarcomeric stress and strain, but the mechanisms by which it does so are unresolved. To gain insight into its mechanosensory function we manipulated single titin molecules with high-resolution optical tweezers. Discrete, stepwise transitions, with rates faster than canonical Ig domain unfolding occurred during stretch at forces as low as $5 \mathrm{pN}$. Multiple mechanisms and molecular regions (PEVK, proximal tandem-lg, N2A) are likely to be involved. The pattern of transitions is sensitive to the history of contractile events. Monte-Carlo simulations of our experimental results predicted that structural transitions begin before the complete extension of the PEVK domain. Highresolution atomic force microscopy (AFM) supported this prediction. Addition of glutamate-rich PEVK domain fragments competitively inhibited the viscoelastic response in both single titin molecules and muscle fibers, indicating that PEVK domain interactions contribute significantly to sarcomere mechanics. Thus, under non-equilibrium conditions across the physiological force range, titin extends by a complex pattern of history-dependent discrete conformational transitions, which, by dynamically exposing ligand-binding sites, could set the stage for the biochemical sensing of the mechanical status of the sarcomere.
\end{abstract}

KEY WORDS: Titin, Mechanosensor, Single-molecule mechanics, Optical tweezers, Monte-Carlo simulation, Atomic force microscopy, PEVK domain

\section{INTRODUCTION}

Mechanical forces are increasingly being acknowledged to influence a wide array of biological processes, to affect the rate of biochemical reactions and to determine the fate of cellular differentiation (Wells, 2013). Mechanobiological phenomena require the sensing of the local mechanical environment, the cellular and molecular mechanisms of which are not well understood. In muscle, it is hypothesized that the giant elastomeric protein titin plays an important role in the

${ }^{1}$ Department of Biophysics and Radiation Biology, Semmelweis University, Tüzoltó u. 37-47, Budapest, H1094 Hungary. ${ }^{2}$ Laboratory of Physiology, BIO c/o Department of Physics, University of Florence, via Sansone 1, 50019 Sesto Fiorentino (FI), Italy. ${ }^{3}$ MTA-SE Molecular Biophysics Research Group, Semmelweis University, Tüzoltó u, 37-47, Budapest H1094, Hungary.

*Present address: Laboratory of Molecular Physiology, NHLBI NIH, Building 50/ 352950 South Drive, Bethesda, MD 20892-8015, USA

§These authors contributed equally to this work.

•Author for correspondence (kellermayer.miklos@med.semmelweis-univ.hu)

Received 12 July 2013; Accepted 18 November 2013 mechanosensory process (Gautel, 2010; Granzier and Labeit, 2005; Krüger and Linke, 2009; Ottenheijm et al., 2011; Voelkel and Linke, 2011). Titin (also known as connectin) forms an integrating filamentous scaffold within the striated muscle sarcomere (Funatsu et al., 1990; Gregorio et al., 1999; Maruyama, 1997; Wang, 1996; Wells, 2013). It spans the middle and the edge of the sarcomere and is tightly bound in the Z-disk, the M-line and the thick filament. It is a linear chain of globular (immunoglobulin, Ig and fibronectin, FN) domains interrupted with unique sequences such as the unstructured PEVK domain (Gautel, 2010; Granzier and Labeit, 2005; Krüger and Linke, 2009; Labeit and Kolmerer, 1995; Ottenheijm et al., 2011; Voelkel and Linke, 2011). Alternative splicing that affects the Iband section results in tissue-specific size isoforms, which differ in the number of Ig domains and the size of the PEVK domain. Whereas nearly 2200 amino acid residues make up the PEVK in the largest skeletal muscle isoform ( $m$. soleus), the N2B cardiac isoform contains merely 163 residues (Bang et al., 2001; Freiburg et al., 2000). Owing to its arrangement within the sarcomere, titin is particularly well localized to sense sarcomeric stress and strain. Therefore, it has been proposed that titin acts as a key mechanosensor in muscle. The complex array of its potential interaction partners, among which even nuclear proteins can be found (Linke, 2007), lend support to the idea. However, the mechanisms behind its mechanosensor function are not known.

A mechanosensor function requires that the molecule be exposed to and reacts to local forces. The response of titin to mechanical forces has been quite extensively studied in singlemolecule experiments (Kellermayer et al., 1997; Rief et al., 1997; Tskhovrebova et al., 1997). The current model of in situ titin extensibility suggests a hierarchical process based on the different persistence length of the various segments and domains within the I-band section of the molecule. At low forces, tandem-Ig regions elongate by straightening of inter-domain linkers (Improta et al., 1996; Linke et al., 1996; Linke et al., 1998b; Trombitás et al., 1998b). At higher forces, the PEVK domain extends continuously as a result of its putative random structure (Li et al., 2002; Linke et al., 1998b; Linke et al., 1998a; Trombitás et al., 1998b). Finally, the N2B unique sequence provides further extensibility, at least in cardiac muscle (Helmes et al., 1999; Linke et al., 1999; Trombitás et al., 2000). Even though globular domains are expected to unfold at increasing passive forces according to the transient-state theory (Rief et al., 1998a), immunolabeling studies have typically failed to demonstrate significant levels of unfolding (Linke, 2000; Trombitás et al., 1998b; Trombitás et al., 2000; Trombitás et al., 2003). Such events might be evoked only beyond physiological circumstances (Minajeva et al., 2001). Mechanosensing requires, in addition, that the properties of titin 
somehow reflect the temporal and spatial features of mechanical exposure. Accordingly, sarcomeric contractile history is expected to influence the mechanical properties of titin. We have previously shown that titin wears out in repeated stretchrelaxation cycles, resulting in mechanical fatigue (Kellermayer et al., 1997; Kellermayer et al., 2001). The molecular detail and the possible role of this process, however, remained elusive. The lack of precise knowledge about the physiologically relevant force range to which a single titin molecule is exposed makes it difficult to pinpoint the potential mechanosensory mechanisms. It is hypothesized that the force per titin molecule in the physiological length range of sarcomeres is small $(\sim 0-20 \mathrm{pN})$ (Anderson and Granzier, 2012; Herzog et al., 2012; Higuchi et al., 1993; Linke and Leake, 2004; Linke et al., 1998a; Liversage et al., 2001). In this force regime, titin is thought to behave as a purely entropic chain devoid of internal interactions. In spite of this, if a relaxed sarcomere is exposed to rapid stretch, passive force decays spectacularly after an initial peak. The mechanisms of this titin-dependent stress-relaxation process are still debated (Minajeva et al., 2001; Trombitás et al., 2003).

Here, we manipulated single molecules of titin by using highresolution optical tweezers in complex nanomechanical scenarios. We found that titin extends, even at very low, physiologically relevant forces, by discrete transitions. The site of the transitions might be allocated to PEVK and to a small number of mechanically weak globular domains in the proximal tandem-Ig or $\mathrm{N} 2 \mathrm{~A}$ region. Although the intra-chain interactions behind the low-force transitions are highly dynamic and are even able to contract titin against mechanical force, the recovery and global pattern of the transitions are strongly influenced by the time- and space-dependent mechanical history of the molecule.

\section{RESULTS}

Single molecules of native, full-length titin purified from rabbit skeletal and cardiac muscles were manipulated in the present work, by using optical tweezers, to resolve mechanically-driven structural changes across physiologically relevant force and temporal scales. Optical tweezers are highly sensitive and robust (Bustamante et al., 2000; Neuman and Nagy, 2008; Smith et al., 2003) and allowed us to manipulate a given titin molecule for time periods up to 20 minutes (Fig. 1A) with sub-picoNewton force resolution. One end of the molecule was captured with a T12 anti-titin-coated latex bead, whereas the other end had a bead coated with the photoreactive crosslinker sulfo-SANPAH. Upon stretching titin with constant velocity, the force rose non-linearly until transitions extending the molecule started to take place. Such transitions began to occur at forces as low as $5 \mathrm{pN}$ (cf. Fig. 2B, inset). To determine what portion of the molecule was captured on average, we calculated the contour length associated with the nonlinear force trace leading up to the first sawtoothshaped transition by fitting with the wormlike-chain equation (Eq. 2; Fig. 1B, left inset). To ensure that data from single titin molecules were analyzed, as opposed to multiple tethers, we identified repetitive sawtooth-shaped transitions at high forces separated by $\sim 30 \mathrm{~nm}$ (Fig. 1B, right inset). Furthermore, we calculated the apparent persistence length of the titin tethers to judge singularity. Fig. 1C shows the distribution of initial contour length mapped onto the size-adjusted domain structure of skeletal muscle titin ( $m$. soleus isoform) (Bang et al., 2001; Freiburg et al., 2000 ). According to this analysis, in $\sim 80 \%$ of the analyzed data, the C-terminal end of the titin tether was within the mechanically active I-band segment.

\section{Characterization of low-force structural transitions}

Force versus extension data obtained in constant-velocity experiments on rabbit $m$. longissimus dorsi titin are shown in Fig. 2. A complete stretch-relaxation cycle is displayed in Fig. 2A. During stretch, transitions that extend the molecule progressively begin appearing below $20 \mathrm{pN}$. The transitions have a similar shape throughout stretch, starting with a rising edge followed by a peak and a falling edge. The rising edge reflects the elasticity of the molecule, whereas the slope of the falling edge corresponds to the stiffness of the optical trap. The peak reflects the instantaneous force in the moment of the chain-lengthening structural transition at the given loading rate (Evans, 2001; Evans and Ritchie, 1997; Evans and Ritchie, 1999). The discrete transitions continued to occur until the end of the stretch halfcycle. However, the spacing between the transitions, which corresponds to the contour-length gain of titin caused by the transition, appears to be different below $\sim 40 \mathrm{pN}$ than above this force. Whereas above $40 \mathrm{pN}$ the transitions are more-or-less evenly spaced by about $30 \mathrm{~nm}$, below $40 \mathrm{pN}$ there is a large variation in contour-length gain, and large steps in contour-length gain also appeared. The relaxation force curve, in contrast to stretch, was always devoid of transitions. Fig. 2B shows a variety of stretch force curves for titin molecules from skeletal muscle. Sawtooth-shaped transitions are systematically present below $5 \mathrm{pN}$ (Fig. 2B inset). Contour-length gain was calculated by fitting the rising half of the force sawtooth with Equation 2 and subtracting from it the contour length of the previous transition (Fig. 2C,D). Transition forces were obtained from the force at the sawtooth peak (Fig. 2D,E). From the scattergram of contourlength gain $\left(\Delta L_{C}\right)$ versus transition force (Fig. 2D), a partitioned distribution emerged. Above $\sim 40 \mathrm{pN}$, a major peak centered at $30 \mathrm{~nm}(31.7 \pm 5.3 \mathrm{~nm}$; mean \pm s.d.) and a minor peak centered at $60 \mathrm{~nm}$ were observed. By contrast, below $\sim 40 \mathrm{pN}$, a major peak between 10 and $80 \mathrm{~nm}$, centered around $30 \mathrm{~nm}$, dominated the wide distribution (Fig. 2D, inset). Persistence length values obtained from the fits varied between 0.9 and $1.9 \mathrm{~nm}$ in case of low-force transitions, and $0.75-0.85 \mathrm{~nm}$ for high-force transitions, and are comparable with earlier measurements (Kellermayer et al., 1997; Leake et al., 2004; Li et al., 2001). The transition force histogram (Fig. 2E) revealed a multimodal distribution with maxima at $12.4 \pm 4.4$ (mean \pm s.d.), 33.9 \pm 9.4 , $61.8 \pm 12.7$ and $75.1 \pm 4.0 \mathrm{pN}$.

The rate of extension at low forces was assessed in constantforce experiments (Fig. 3). Titin was stretched to a predetermined set force value, which induced an initial rapid extension of the molecule to maintain the force level constant. Even at low clamp forces, the molecule continued extending in well discernible steps (Fig. 3A). We varied the clamp force to gain access to the spontaneous transition rate (Fig. 3B). The time-dependent extension traces were fitted with double-exponential functions. The extension-step histogram (Fig. 3B, inset) revealed a multimodal distribution with major peaks at around 11, 25 and $39 \mathrm{~nm}$. From the load-dependent rate constants (Fig. 3C), we calculated the spontaneous rates of extension based on Equation 3: for the fast component we obtained a spontaneous rate $\left(k_{0}\right)$ of 0.044 second $^{-1}$, and for the slow component, $k_{0}$ was 0.013 second $^{-1}$. The distance, along the reaction coordinate, from the contracted to the transition state $(\Delta x)$ was 0.36 and $0.18 \mathrm{~nm}$ for the fast and slow components, respectively. Notably, most of the extension versus time traces contained backward steps, which was particularly evident in traces at low $(<20 \mathrm{pN})$ clamp forces (Fig. 3B). Fig. 3D shows a magnified trace that 


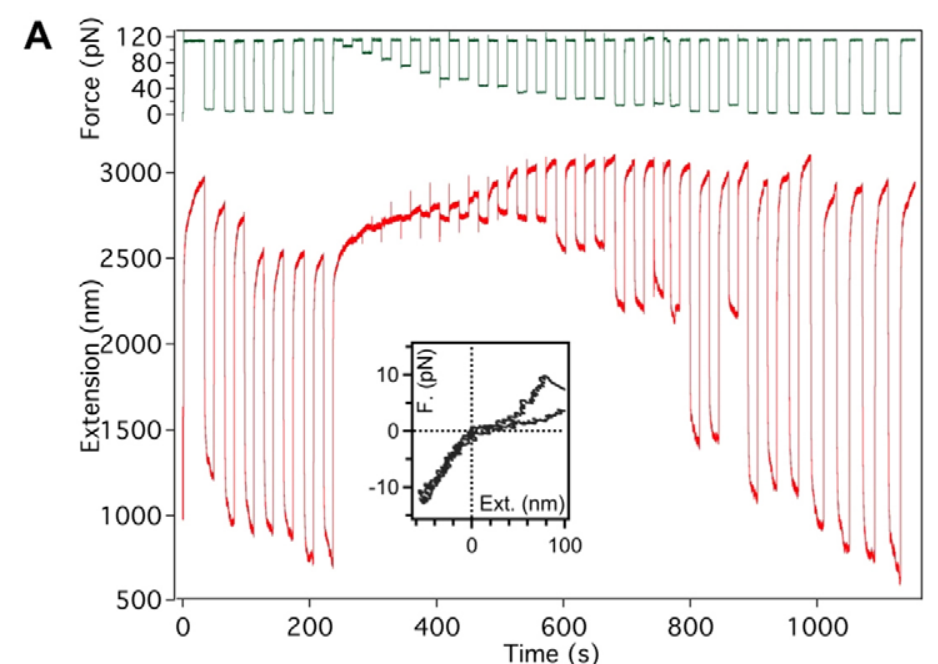

B
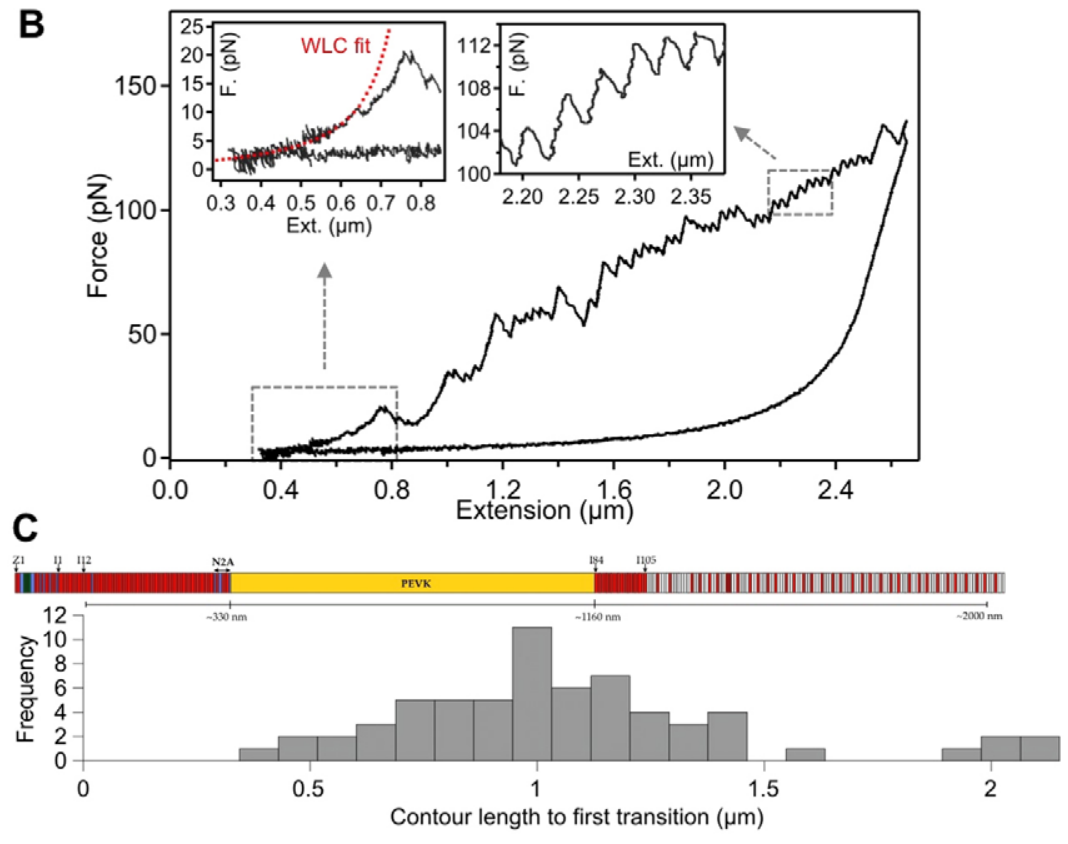

Fig. 1. Force spectroscopy of titin with optical tweezers. (A) Force-clamp spectrum displaying force and molecular extension as a function of time, demonstrating the robustness of the method. A molecule of skeletal muscle titin was stretched and relaxed to 115 and $5 \mathrm{pN}$ of constant force, respectively, in 36 cycles. The molecule was stable for 20 minutes. A quasi force ramp was introduced between 250 and 800 seconds. Inset shows force versus extension curve of a mechanical cycle under velocity clamp, during which the beads at the end of the titin molecule were pressed against each other. (B) Force versus extension curve of skeletal muscle titin under velocity clamp, with wormlike-chain fit (WLC, see Eq. 2) to the data leading to the first transition (left inset). Force curves containing repetitive sawtooth pattern with peaks $\sim 30 \mathrm{~nm}$ apart (right inset) at forces typically above $50 \mathrm{pN}$ were selected for analysis, because these are hallmarks of single-molecule behavior (Rief et al., 1997; Tskhovrebova et al., 1997). (C) Distribution of the contour lengths, associated with the first transition in skeletal muscle titin manipulation experiments, mapped approximately onto the domain structure of full-length skeletal titin $(\mathrm{m}$. soleus isoform) (Bang et al., 2001; Freiburg et al., 2000). The tandem globular domain regions were assumed to be completely folded but straightened to a length of $5 \mathrm{~nm} /$ domain $(4 \mathrm{~nm}$ domain length plus $1 \mathrm{~nm}$ linker). The PEVK domain was assumed to be completely straightened $(830 \mathrm{~nm})$. contains several backward transitions, which result in contraction. Interestingly, the backward, contractile transitions were often less abrupt than the forward steps, suggesting that the contraction, by structural transitions, of titin is a slower process that occurs through intermediate states. Altogether, the second timescale forward (extending) and backward (contracting) steps set the endto-end length of the molecule. To investigate the temporal dynamics of the low-force transitions further, titin was stretched at constant velocity in successive mechanical cycles (Fig. 4A). In stretch-relaxation cycles repeated immediately after one another, the force hysteresis did not recover completely, and stretch-force traces shifted to higher extensions. Although the stretch transitions appeared below $40 \mathrm{pN}$ even in the second mechanical cycle, their number and the resultant gain in contour length became significantly smaller. Thus, a major cause of titin molecular fatigue (Kellermayer et al., 2001) is the disappearance of the discrete low-force transitions.

\section{Possible site of low-force structural transitions in titin}

To investigate which regions of titin are responsible for the lowforce transitions, we conducted three different types of experiments in which we: (1) reduced the ionic strength; (2) treated titin with an anti-PEVK antibody (9D10); and (3) made measurements on cardiac titin, which contains a PEVK domain that is less than $10 \%$ of the size of that in the skeletal isoform. Supplementary material Fig. S2 shows an example of the mechanical cycles on titin at reduced ionic strength $(100 \mathrm{mM}$ $\mathrm{KCl}$ ). The rationale behind this experiment was our previous observation that the effective persistence length of the PEVK domain can be tuned with ionic strength (Nagy et al., 2005): the smaller the ionic strength, the greater the persistence length. Our results indicate that the majority of small- $\Delta L_{C}$ lowforce transitions disappeared at the reduced ionic strength, but molecular fatigue was still present. Notably, large- $\Delta L_{C}$ transitions (in the order of $100 \mathrm{~nm}$ ) often persisted (supplementary material Fig. S2, inset). Supplementary material Fig. S3 shows experiments in which the manipulated molecule was treated with the 9D10 anti-PEVK antibody, which binds along the entire length of the domain (Trombitás et al., 1998a). We observed stretch force curves devoid of major transitions, and molecular fatigue was present in immediately successive mechanical cycles (supplementary material Fig. S3, top trace). 

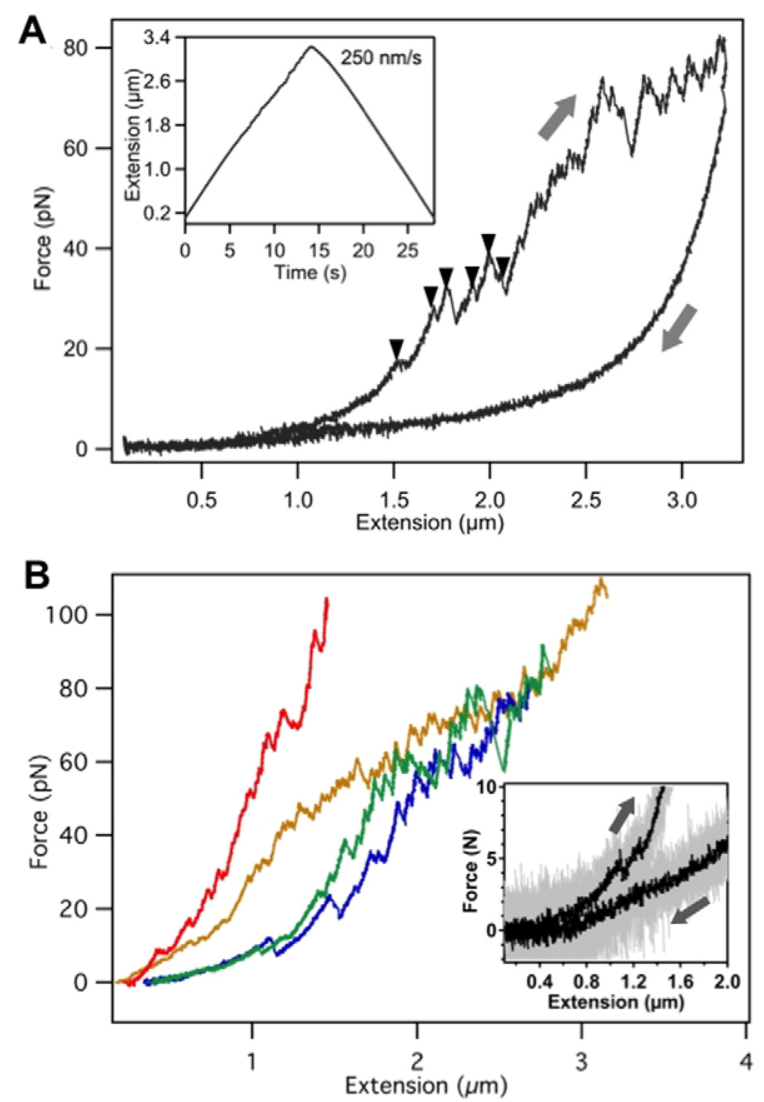
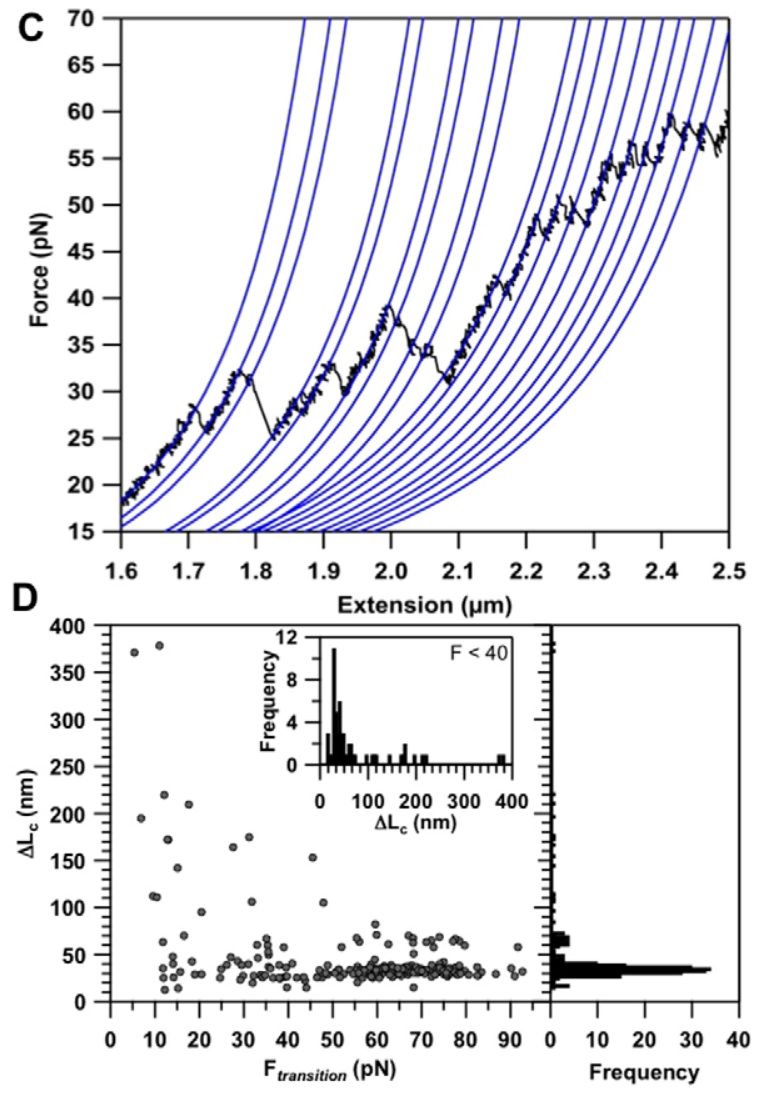

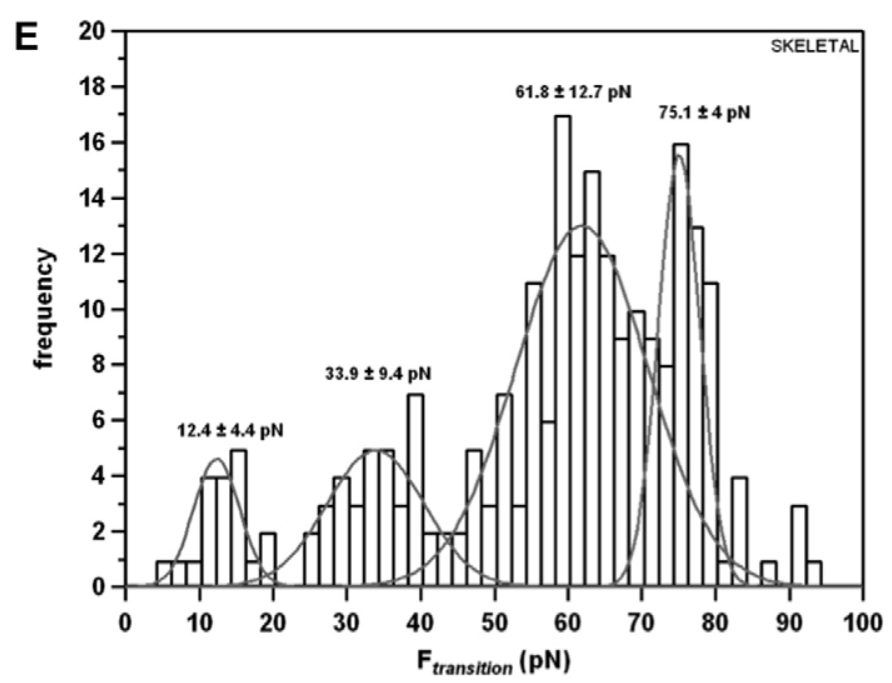

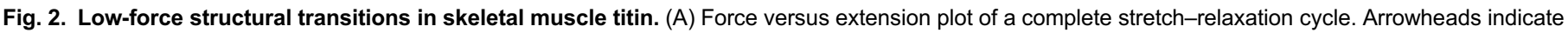

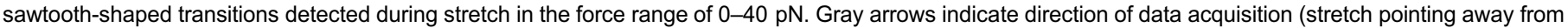
and relaxation towards zero extension). Inset shows mechanical protocol in an extension versus time function. (B) Ensemble of stretch curves obtained on different titin molecules displaying multiple low-force transitions. Inset shows an example of transitions at $\sim 5 \mathrm{pN}$ in filtered (smoothed by passing a 10-point

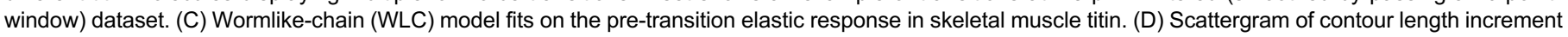
$\left(\Delta L_{c}\right)$ versus instantaneous transition force $\left(F_{\text {transition }}\right)$. Inset, distribution of $\Delta L_{c}$ at forces $<40 \mathrm{pN}$. (E) Distribution of the transition forces. Gaussian fits were made on the local modes. Mean transition force and s.d. are indicated above the peaks.

However, force hysteresis essentially recovered if short 10 second pause periods were inserted between mechanical cycles (supplementary material Fig. S3, bottom trace). To completely exclude PEVK, we manipulated titin by grabbing it with beads coated with the T12 and 9D10 antibodies. In these experiments, we thus manipulated the proximal tandem-Ig and N2A regions of titin. The initial contour length of the chain was only $120 \mathrm{~nm}$, and the force trace was dominated by transitions that extended the contour length of the molecule by $30 \mathrm{~nm}$ on average (Fig. 4B), suggesting that Ig-domain unfolding exclusively determined the nanomechanical behavior of this titin tether. Interestingly, three domains unfolded at forces below $40 \mathrm{~N}$, and this was repeatable 

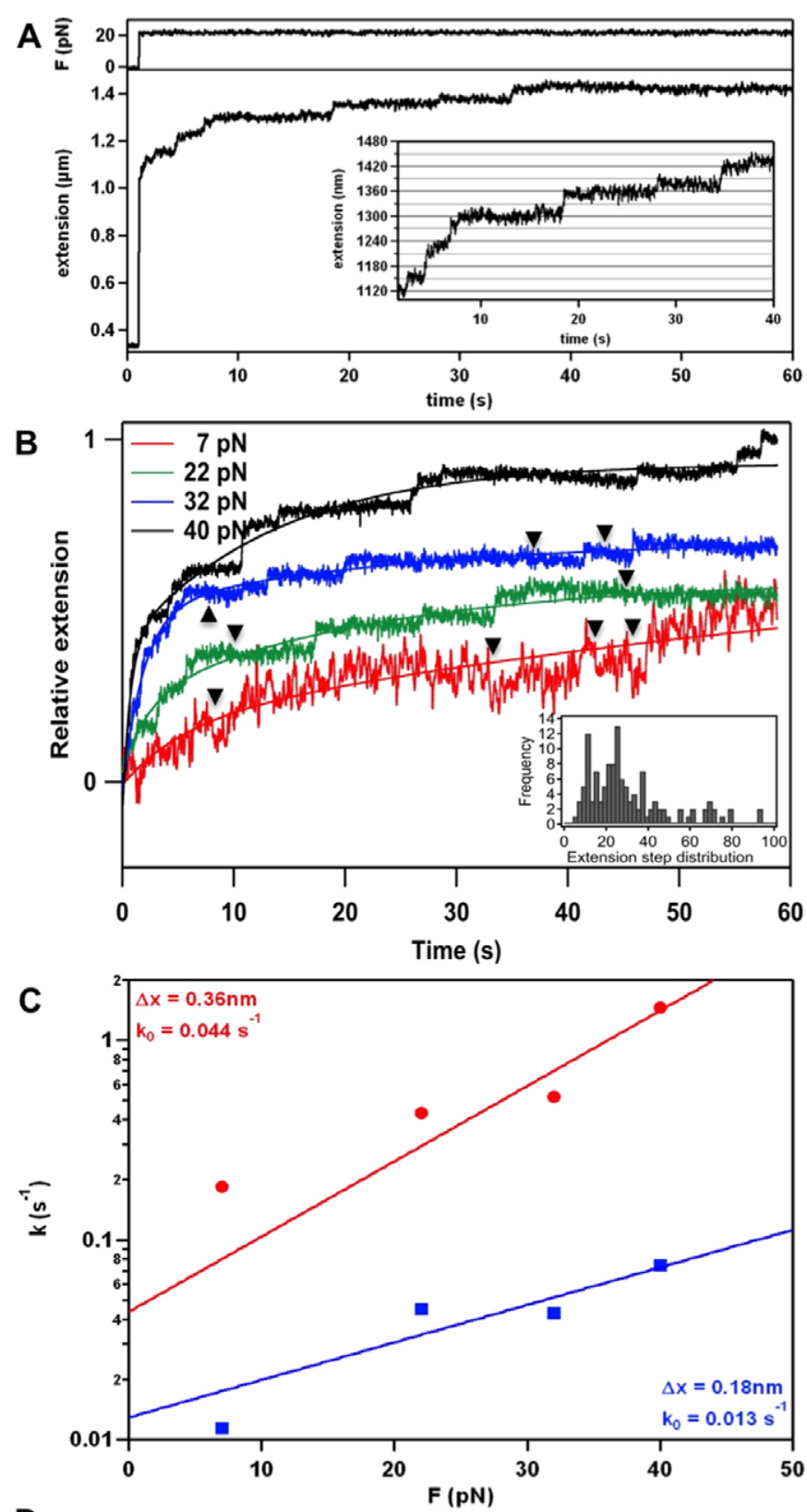

D

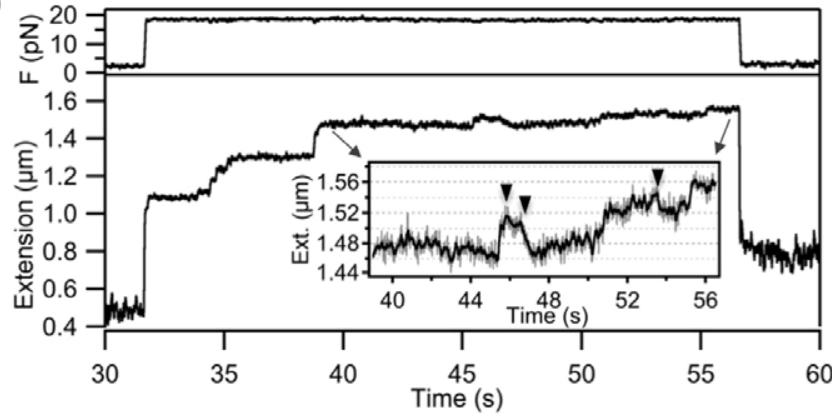

in successive mechanical cycles. Force hysteresis completely recovered during the 10 -second pause in the contracted state between the mechanical cycles.

To explore the effect of reducing the size of PEVK, we manipulated rabbit cardiac titin (Fig. 5). Similar to the skeletal isoform, discrete transitions were observed in the stretch force
Fig. 3. Constant-force stretching of skeletal muscle titin. (A) Example of an extension versus time plot collected for 60 seconds. Inset is a magnified view of step-wise changes in extension. (B) Double-exponential fit of the extension versus time functions at different clamp forces (7-40 pN). Arrowheads indicate reverse contractile transitions. Inset shows distribution of extension steps. (C) Plot of the fast (red) and slow (blue) rate constants as a function of clamp force. Data were fitted with equation 3. (D) Reverse (contractile) steps observed during force-clamp spectroscopy. Clamp force, $18 \mathrm{pN}$. Inset shows a magnified view of the extension versus time trace. Arrowheads indicate contractile transitions.

traces even at forces below $40 \mathrm{pN}$ (Fig. 5A,B). Persistence length varied between 1.2 and $1.8 \mathrm{~nm}$ in the case of low-force transitions and between 0.7 and $1.0 \mathrm{~nm}$ for high-force transitions. The distribution of contour-length gain contained a major peak centered at $30 \mathrm{~nm}(28.6 \pm 4.5 \mathrm{~nm})$ and a minor peak at $60 \mathrm{~nm}$, and $\Delta L_{C}$ was broadly distributed below $40 \mathrm{pN}$ (Fig. 5C). However, the overall $\Delta L_{C}$ distribution was much narrower than in the case of the skeletal isoform. Force maxima were present in the transition-force histogram (Fig. 5D): $19.5 \pm 6.6$ (mean \pm s.d.), $33.6 \pm 5.4,47.4 \pm 7.6$ and $64.6 \pm 7.0 \mathrm{pN}$.

\section{Effect of mechanical history on low-force transitions}

To investigate the potential biological significance of the lowforce transitions and their role in titin mechanical fatigue, we carried out stretch and relaxation experiments in complex patterns. First, titin was stretched in immediately successive mechanical cycles at increasing stretch velocities (supplementary material Fig. S4). The loss of hysteresis recovery progressively increased with increasing stretch rates, and upon reaching $1000 \mathrm{~nm} /$ second the hysteresis nearly completely disappeared in the second mechanical cycle. Force hysteresis could be recovered upon resting the molecule in the contracted state (supplementary material Fig. S5; Fig. 6A). Whereas hysteresis recovered only partially during a 10-second pause (supplementary material Fig. S5 top), it recovered completely after 20 seconds (supplementary material Fig. S5 bottom), and after 30 seconds it even exceeded that seen in the first cycle (Fig. 6A). We observed a specific tendency in the gradual return of the force hysteresis: after short periods of resting time the low-force transitions returned, and greater periods of rest were necessary for the recovery of transitions at high forces (supplementary material Fig. S5). Thus, the time spent in a distinct contraction state significantly influences the force pattern in the next mechanical cycle. To investigate how the contraction state influences titin mechanical fatigue, we stretched a single molecule with constant, $250 \mathrm{~nm} /$ second velocity to an extension of $2.8 \mu \mathrm{m}$, then rapidly quenched the extension to different end-to-end lengths: 614 (supplementary material Fig. S6, top); 743 (supplementary material Fig. S6, bottom) and $941 \mathrm{~nm}$ (Fig. 6B) and paused for 20 seconds. The average force acting upon the molecule was similar and almost negligible in each of the nanomechanical tests $(0.27 \pm 0.52$, $0.81 \pm 0.46$ and $1.02 \pm 0.40 \mathrm{pN})$. Subsequently, the molecule was stretched in a complete mechanical cycle with $250 \mathrm{~nm} /$ second velocity (supplementary material Fig. S6B). Upon increasing the resting end-to-end length of the molecule, the probability of recovering the force hysteresis gradually decreased, and the initial stretch force curve became progressively shifted to increasing extensions. The overall pattern of the high-force transitions $(>40 \mathrm{pN})$, judged from the sequence of the sawtooth force peaks, however, remained similar while being shifted right (to high extensions). Thus, most of the globular domains unfolded during the first mechanical cycle refolded during the 20 -second rest 
A
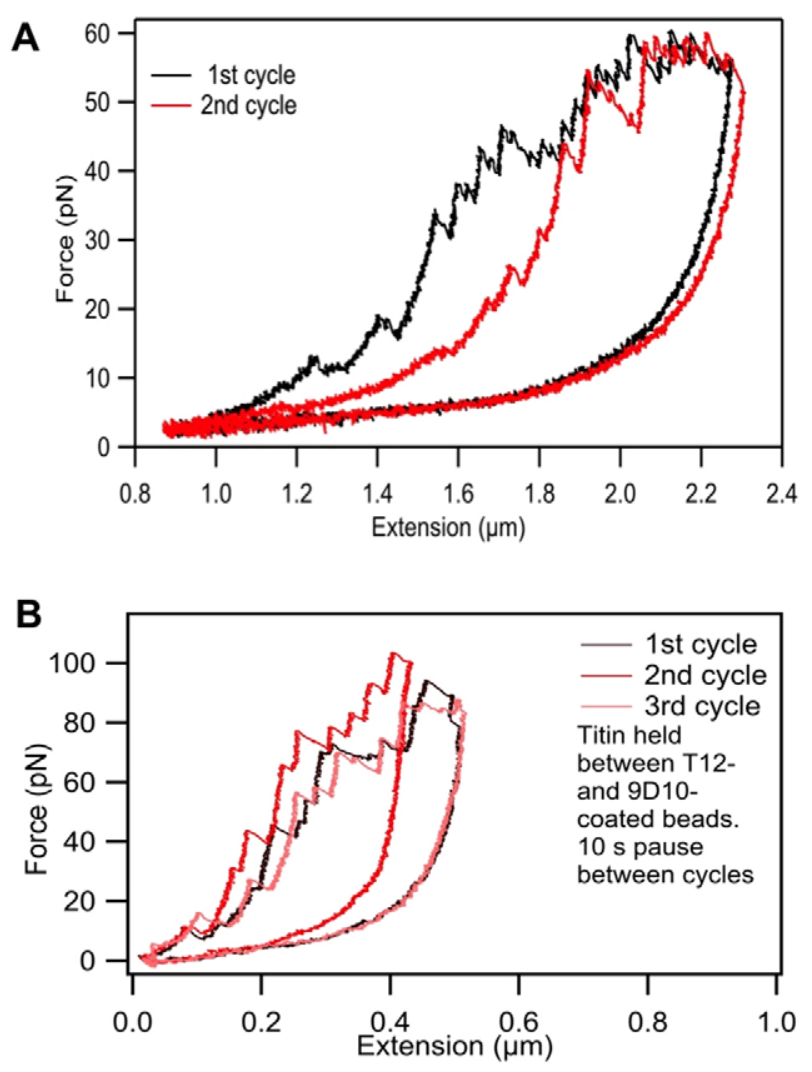

Fig. 4. Testing the long-timescale mechanical behavior and contribution of the PEVK domain. (A) Change in the force versus extension plot in subsequent mechanical cycles. Constant velocity mode at $250 \mathrm{~nm} /$ second with no pause in between the mechanical cycles. (B) Stretching skeletal muscle titin by grabbing it with T12 and 9D10 antibody-coated beads. Force versus extension plots obtained in successive stretch-relaxation cycles with a 10 second rest phase in between.

period in all cases, whereas the intramolecular interactions behind the low-force transitions failed to be re-established if the end-toend length of titin was increased, and the stretch curve of the second mechanical cycle was highly sensitive to the resting extension of the titin molecule.

\section{DISCUSSION}

\section{Complex optical tweezers force spectroscopy of titin}

We mechanically manipulated individual titin molecules from rabbit longissimus dorsi and cardiac muscles by exposing them to complex tests with high-resolution optical tweezers. Force spectroscopy provides unprecedented insight into the molecular detail of important biochemical and biophysical processes (Fisher et al., 2000; Neuman and Nagy, 2008; Rief and Grubmüller, 2002). The most frequently used methods are based on the atomic force microscope (AFM). However, optical tweezers provide several advantages over AFM particularly in the case of long chain-like molecules such as native, full-length titin (Kellermayer et al., 1997; Kellermayer et al., 1998; Kellermayer et al., 2000; Leake et al., 2004; Tskhovrebova et al., 1997): (1) multiple consecutive mechanical cycles can be carried out (Fig. 1A); (2) complex nanomechanical scenarios can be executed (Fig. 6; supplementary material Figs S4-S6); (3) solute effects can be studied on the same molecule (Fig. 4; supplementary material Figs S2,S3); (4) the sensitivity of the method to probe-surface interactions is smaller than in AFM (Fig. 1A, inset) and can be reduced with conventional blocking agents (e.g. BSA); (5) the smaller probe stiffness $(0.2 \mathrm{pN} / \mathrm{nm})$ provides greater force sensitivity and a gentle action; $(6)$ the force range ( $\sim$ three orders of magnitude, $0.5-150 \mathrm{pN}$ ) is on par with that of AFM but shifted to smaller forces by two orders of magnitude; and (7) the extension range is several tens of micrometers compared with the few hundred nanometers typical in usual AFM. Thus, optical tweezers force spectroscopy is particularly well suited to investigate the molecular detail behind the extensibility and force-driven structural changes of the giant titin molecule.

To stretch titin, its N-terminal end was captured with a latex bead coated with the T12 antibody that binds to the I12 domain (Fürst et al., 1988). This antibody has been successfully used previously (Kellermayer et al., 1997). Towards the C-terminus, titin was captured with a bead coated with the photoreactive crosslinker sulfo-SANPAH (Kellermayer et al., 1997). Although this crosslinker provides a stable attachment, it lacks sequence specificity. Therefore, the exact contour length of the captured titin tether cannot be established a priori. To estimate the contour length of the tether a posteriori, we fitted the wormlike-chain equation (Eq. 2) on the initial non-linear portion of the force traces leading up to the first detectable transition (Fig. 1B, left inset). The obtained contour-length distribution was mapped onto the domain layout of skeletal muscle titin (Fig. 1C) (Bang et al., 2001; Freiburg et al., 2000). In assembling the distance-corrected domain-layout map of titin we assumed that all globular domains are folded and the PEVK is completely extended, because this is the assumed scenario of titin extensibility at low forces (Linke et al., 1999; Trombitás et al., 1998b). Considering that the A-band-I-band junction is located at the I105 domain (Bang et al., 2001; Freiburg et al., 2000), in $80 \%$ of the mechanical experiments we manipulated a titin tether that mapped within the physiologically extensible, I-band segment of titin. However, we might have underestimated the contour length of the relevant titin-domain layout for a number of reasons: we used the available human soleus isoform domain structure (Bang et al., 2001; Freiburg et al., 2000), which is longer than the rabbit longissimus dorsi isoform, and there are probably intradomain interactions and transient structures present in the PEVK domain, which result in a shortened effective contour length. However, because we do not have control over whether all of the globular domains are indeed in the folded state, it is a fair approximation that the majority of the data was obtained in I-band titin tethers.

\section{Discrete low-force transitions of the titin molecule}

In velocity-clamp $(250 \mathrm{~nm} /$ second $)$ experiments, sawtoothshaped transitions appeared during stretch, which extended the contour length of the molecule in a step-wise manner (Fig. 1B and Fig. 2). Above $\sim 40 \mathrm{pN}$, the sawtooth-shaped transitions extend the molecule by $\sim 30 \mathrm{~nm}$ on average in both skeletal and cardiac titin, therefore these transitions are identified as globulardomain unfolding (Rief et al., 1997; Tskhovrebova et al., 1997). The $60 \mathrm{~nm}$ peak in the $\Delta L_{c}$ histogram most likely corresponds to the unfolding of two domains within a time period of 1 millisecond, which is beyond the time resolution of our instrument. Notably, at the stretch rate used $(250 \mathrm{~nm} / \mathrm{s})$, the various recombinant globular domains of titin investigated so far unfold at forces well above $100 \mathrm{pN}$ (Grama et al., 2005), therefore, mechanically unstable domains are present in the manipulated titin section. It has been previously shown that the FN-type domains, present in the A-band section of the molecule, are mechanically less stable than the Ig-type, therefore they 

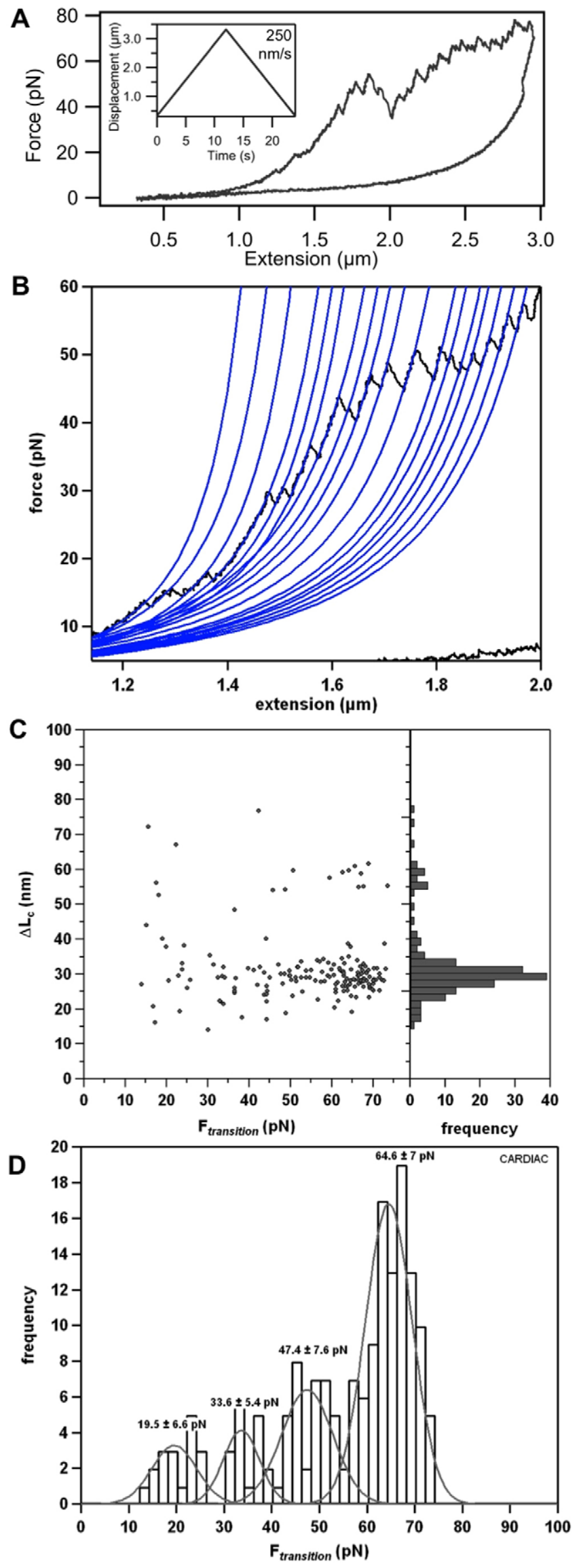

Fig. 5. Manipulation of native rabbit cardiac titin. (A) Example of a force versus extension plot of a complete stretch-relaxation cycle of cardiac titin. Inset shows the experiment in terms of the displacement of one end of the molecule as a function of time. (B) Wormlike-chain (WLC) fits to the rising half of the stretch transitions. (C) Scatter plot of contour length displacement $\left(\Delta L_{c}\right)$ as a function of transition force $\left(F_{\text {transition }}\right)$. (D) Distribution of transition force.

unfold at lower forces (Rief et al., 1998b). There might indeed be a few A-band FN domains present in some of the longer titin tethers manipulated in our work. However, the large number of unfolding transitions below $100 \mathrm{pN}$ observed here within single force traces cannot be explained solely by their presence. Rather, it is likely that mechanically unstable domains are also present within the tandem-Ig segments. Experiments in which only the proximal tandem-Ig region of titin was manipulated (Fig. 4B) demonstrate that this segment of the molecule indeed contains a few (two to three) domains that unfold below $40 \mathrm{pN}$. The precise allocation of these mechanically unstable domains to the sequence and domain structure of titin requires further experimentation.

We observed a high frequency of discrete transitions even below $\sim 40 \mathrm{pN}$ (Fig. 2). Some of the lowest force transitions occurred at around $5 \mathrm{pN}$. In the sub-40-pN transition-force range, the $\Delta L_{c}$ distribution became wide (ten to several hundred $\mathrm{nm}$ ), indicating that the increment steps of contour length are not solely due to the unfolding of globular domains, but additional forcebearing conformations and interactions between remote, structurally less specific binding sites of the titin chain also contribute to the mechanical behavior. Previously, we have shown that in the low-force regime $(<20 \mathrm{pN})$, molecular fatigue occurs in titin (Kellermayer et al., 2001), which is probably caused by the rupture of intrachain bonds within the PEVK domain. It is plausible that PEVK contributes significantly to the appearance of the discrete transitions, even though previously we have not been able to detect such steps, probably because of low force and time resolution. During molecular fatigue, the rupture of intrachain bonds extends the contour length of titin during stretch, but the bonds are unable to recover on a short time scale; therefore, the process manifests in a progressive shift of the stretch force curve towards increasing extensions and a corresponding reduction in force hysteresis. Our current highresolution measurements also revealed the molecular fatigue phenomenon (Fig. 4A) and indicate that both regularly and irregularly spaced sawtooth-shaped force transitions, corresponding to globular-domain unfolding and conceivably to bond rupture or unfolding of transient structures (Huber et al., 2012; Ma and Wang, 2003) within the PEVK domain, respectively, contribute to the process. To analyze the role of PEVK in the low-force discrete transitions and molecular fatigue, we used several distinct experimental strategies: (1) ionic strength was varied by changing $\mathrm{KCl}$ concentration; (2) the PEVK domain was rendered inactive with the 9D10 antibody; and (3) we used cardiac titin, which contains a PEVK domain with a size $10 \%$ that of the skeletal isoform (Labeit and Kolmerer, 1995). Upon reducing $\mathrm{KCl}$ concentration from $200 \mathrm{mM}$ to $100 \mathrm{mM}$, the lowforce regime became devoid of low- $\Delta L_{C}$ discrete transitions, but molecular fatigue persisted (supplementary material Fig. S2). Reduction of ionic strength electrostatically stiffens the PEVK domain (Nagy et al., 2005), which probably prevents the formation of short-range interactions within the domain. If PEVK was inactivated with the 9D10 antibody, which binds 
A

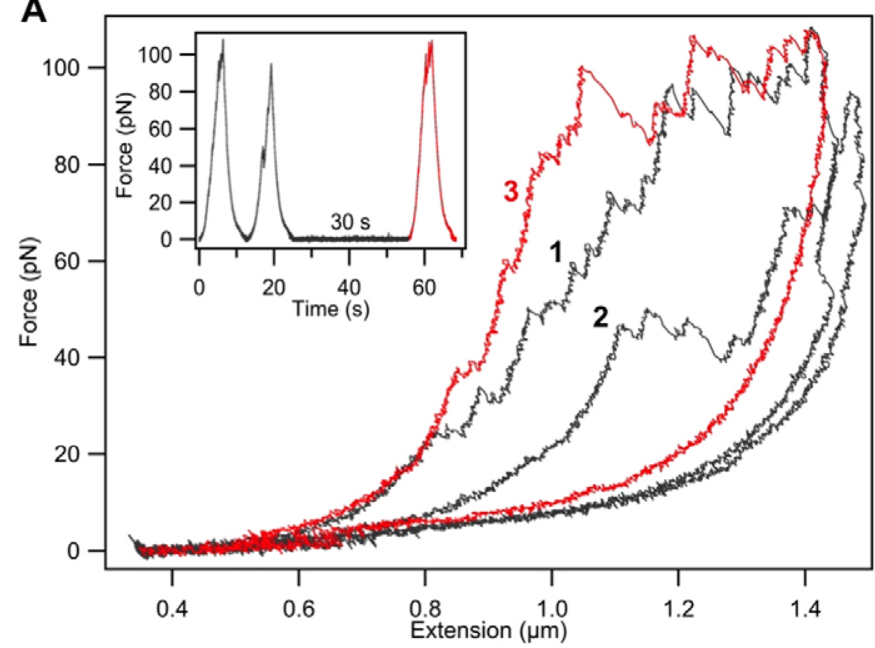

B

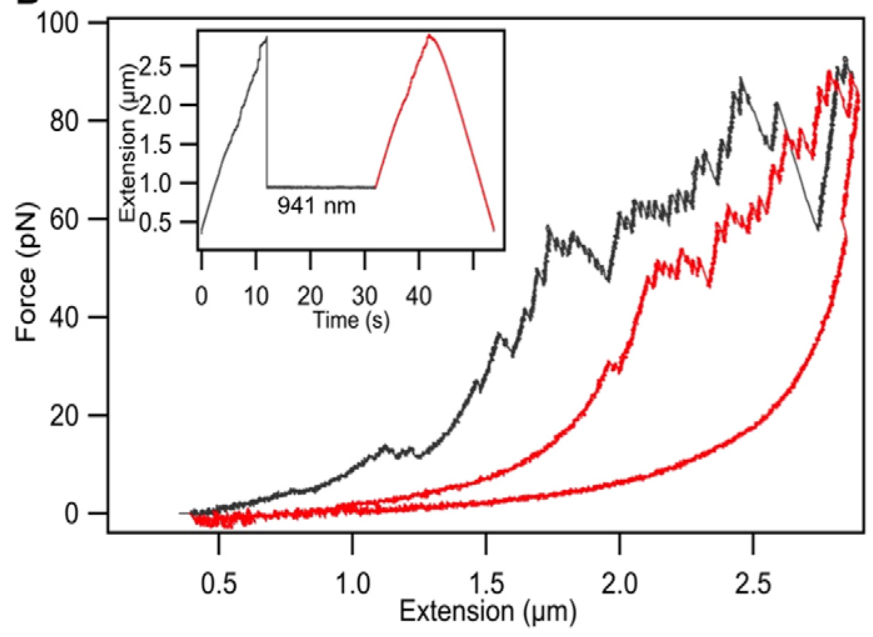

Fig. 6. Complex mechanical tests on titin. (A) Effect of slack pause time on titin fatigue. A skeletal muscle titin molecule was manipulated in two immediately successive stretch-relaxation cycles (stretch rate $250 \mathrm{~nm} /$ second) followed by a rest period of 30 seconds, then by a third, monitoring stretch-relaxation cycle. The inset shows the layout of the mechanical procedure in terms of force as a function of time. (B) Effect of contractile status on titin fatigue. The skeletal muscle titin molecule was first stretched, at a constant rate of $250 \mathrm{~nm} / \mathrm{second}$, to an extension of $\sim 2.8 \mu \mathrm{m}$, then the extension was rapidly quenched for 20 seconds to $941 \mathrm{~nm}$, then a complete stretch-relaxation cycle was carried out. The inset shows the layout of the mechanical procedure in terms of extension as a function of time.

along the entire length of the domain (Trombitás et al., 1998a), the stretch curve again became devoid of low-force transitions, yet molecular fatigue was still present. In further support of the role of PEVK in the low-force discrete transitions, when cardiac titin was manipulated the $\Delta L_{c}$ distribution in the sub-40-pN force range became highly reduced (Fig. $5 \mathrm{C}$ ), in accordance with the smaller size of the domain in this isoform. Previously, step-like transitions in the PEVK domain have been reported in experiments with optical tweezers on skeletal muscle titin held between beads coated with anti-N2A and anti-I84-86 antibodies, which demarcate the boundaries of the PEVK domain along the titin molecule (Leake et al., 2004). Even though our results are not fully comparable with that study, because of the somewhat different experimental arrangement, different force levels and the opposite ionic-strength effects, the $\Delta L_{c}$ distribution reported by
Leake and colleagues displays a striking similarity to the extension distribution measured at low forces in our present work (Fig. 3B, inset). Accordingly, the $\sim 11,25$ and $39 \mathrm{~nm}$ steps might correspond to unitary length changes that accompany the force-driven conformational changes or bond-rupture events in PEVK. We hypothesize that two types of structural transitions occur in PEVK in response to stretch: unfolding of low-energy conformations (polyproline helices, $\beta$-turns) (Huber et al., 2012; $\mathrm{Ma}$ and Wang, 2003) present in the 28-mer repeat units (Greaser, 2001) and rupture of electrostatic interactions between more distant, oppositely charged regions. Whereas the re-establishment of the former is inhibited at low ionic strength as a result of electrostatic stiffening, the latter is present even at low ionic strengths (supplementary material Fig. S2). Altogether, in the low-force regime mechanics of the titin molecule, both the stepwise extension of the PEVK and the unfolding of low-stability globular domains are manifest, and in a given force response, the structural transitions caused by either of these processes follow each other randomly.

The force-driven structural transitions in titin contribute to its viscoelasticity. Accordingly, the molecule - hence the relevant muscle structures such as the myofibril and the muscle fiber displays stress relaxation (Anderson and Granzier, 2012). The exact molecular mechanisms behind sarcomeric stress relaxation are not known. Some observations indicate progressive domain unfolding (Minajeva et al., 2001), but others implicate the PEVK domain (Trombitás et al., 2003). Here, we propose that both globular domain unfolding and structural transitions within the PEVK contribute to relaxation of sarcomeric stress. Because immunoelectron microscopic observations suggest that under equilibrium conditions the extensibility of tandem-Ig regions due to unfolding remains low, it remains a question how the globulardomain unfolding mechanism can be reconciled with our suggestion. Conceivably, mechanically unfolded domains remain in a compact but compliant state (Cecconi et al., 2005; Elms et al., 2012; Garcia-Manyes et al., 2009), which might not be distinguishable from the folded state with immunoelectron microscopy techniques.

The physiological relevance of the low-force structural transitions might be understood by extrapolating the sarcomeric passive-force range to a single titin molecule. Previous estimates for the physiological maximum force per titin vary between 5 and $20 \mathrm{pN}$ (Anderson and Granzier, 2012; Herzog et al., 2012; Higuchi et al., 1993; Linke and Leake, 2004; Linke et al., 1998a; Liversage et al., 2001). Even if the lowest forces per titin molecule are considered, the mechanically-driven transitions reported here are important under physiological conditions. Furthermore, it is important to note that the forces obtained in muscle preparations (myofibrils, myocytes or fibers) are average equilibrium forces. Thus, the titin molecules are likely to be mechanically relaxed and in a state in which the low-stability interactions are already broken.

The rate of the titin-lengthening transitions, obtained by extrapolation of force-clamp data, is an order of magnitude greater than that measured for the I27 domain (Garcia-Manyes et al., 2007), further supporting the notion that the corresponding interactions are unstable. Double-exponential function gave a better fit than the single exponential, suggesting that two processes are present. Possibly, one corresponds to the unfolding of the low-stability domains and the other to the intra-PEVK transitions. The observation of several modes in the transition-force histograms (Fig. 2E and Fig. 5D) lends further 
support to the idea that multiple interactions are behind the transitions. We also found that reverse or contractile steps take place against force. The presence of reverse steps suggests that a balance of contraction-extension transitions dynamically adjusts the contour length of titin across physiological force and temporal scales. The exact nature of the reverse steps, however, is unclear. The few high-resolution records of the reverse steps indicate that the process might proceed via small substeps (Fig. 3D), suggesting that the contraction mechanism is not the exact opposite of the extension step.

\section{Titin as mechanosensor}

The force versus extension response of titin reflects the recent history of the mechanical conditions to which the molecule has been exposed. By sensing the mechanical environment and providing a suitably modified force response, titin fulfils a mechanosensor function. The mechanical parameters that influence mechanical response are force, space and time. Accordingly, the stretch-force curve of titin is sensitive to prior loads and loading rates, the end-to-end distance at which it was held and the time spent in a given configuration (Fig. 6; supplementary material Fig. S4-S6). The response function is a history-dependent change in the effective contour length, which manifests in a shift of the force trace along the extension axis. Progressive shift towards increasing or decreasing extensions corresponds to molecular fatigue (Kellermayer et al., 1997; Kellermayer et al., 2001) or mechanical recovery, respectively. Our results indicate that a dual molecular mechanism is responsible for the mechanosensitive response: the PEVK domain and a few unstable domains in the proximal tandem-Ig region. Upon stretching PEVK, its transient structures and electrostatic interactions break under load in a stochastic manner, resulting in step-wise contour-length increments; hence the appearance of sawtooth-shaped peaks in the force spectrum. The intradomain interactions could be re-established by a self-zippering mechanism, whereby neighboring, then progressively more distant regions of the chain contact and bind each other, depending on the status of the contraction. In addition to PEVK, globular domains also appear to participate in the mechanosensory function. On one hand, there are a few mechanically unstable domains in the proximal tandem-Ig or N2A segment, which unfold at low forces (Fig. 4B). The sequence identity of these domains is yet to be uncovered. On the other hand, additional domains, unfolding at greater forces, contribute to the molecular fatigue mechanism by failing to refold in time before the subsequent mechanical cycle (Figs 4,6; supplementary material Figs S2-S6).

To test the above mechanosensor mechanism of titin and investigate its relevance in a cellular context, we carried out simulations, high-resolution AFM measurements on overstretched titin molecules and mechanics measurements of single muscle fibers. Monte-Carlo simulation based on our simple dualistic model recovered the essential features of our findings (Fig. 7A; supplementary material Figs S7-S9). In the force spectrum, globular-domain unfolding and intra-PEVK events are intermixed. Thus, the model predicts that the unfolding of globular domains occurs even before complete PEVK extension. To test for this possibility, we stretched titin molecules with receding meniscus (Tskhovrebova and Trinick, 1997; Tskhovrebova and Trinick, 2001) and visualized their highresolution structure with AFM (Fig. 7B) (Mártonfalvi and Kellermayer, 2014). In only $2 \%$ of the analyzed molecules
A
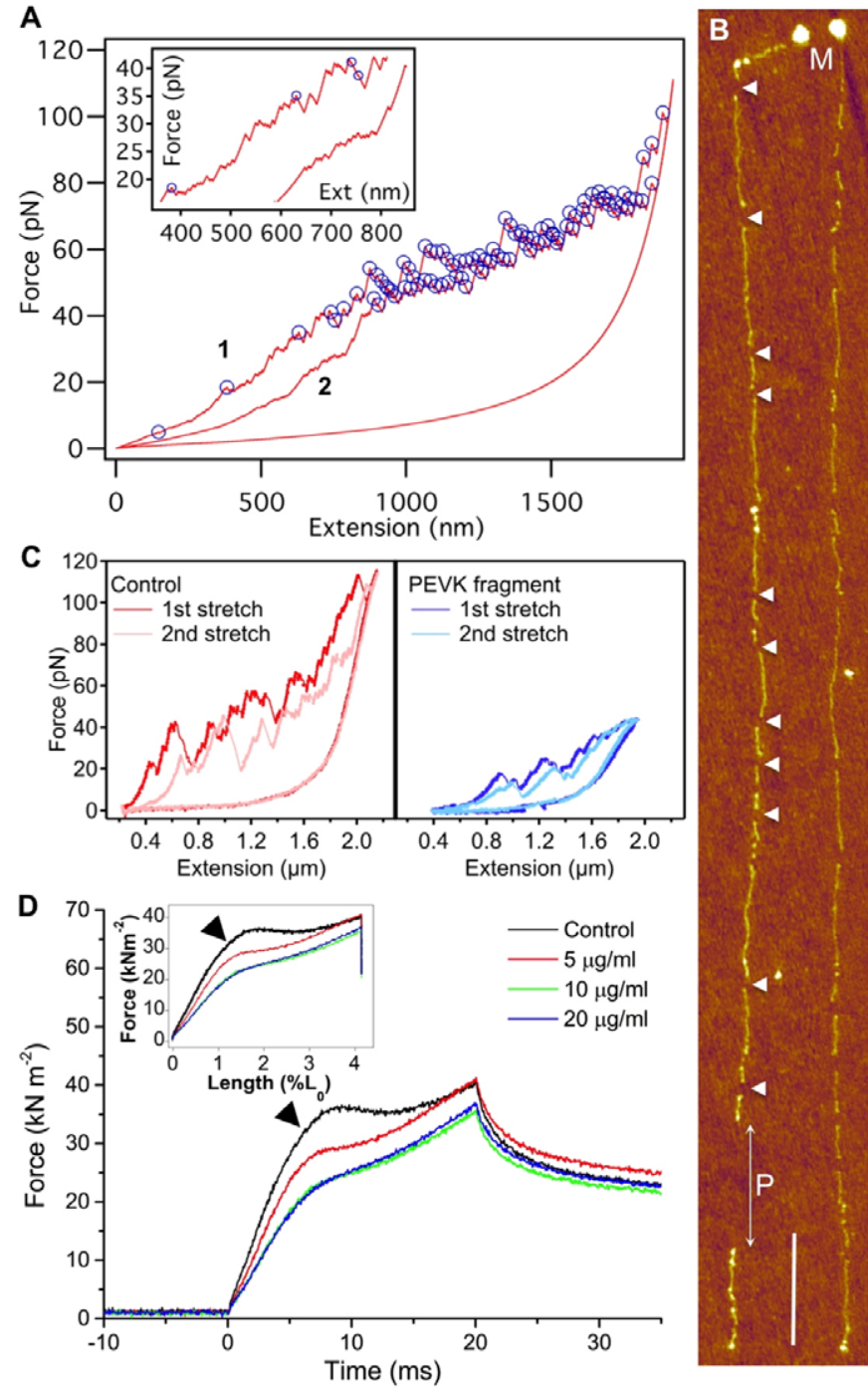

Fig. 7. Simulation high-resolution imaging and fiber testing of titin lowforce transitions. (A) Monte-Carlo simulation of titin behavior and AFMbased test of the model prediction. Force versus extension plot of a simulated molecule stretched at a rate of $250 \mathrm{~nm} /$ second in two consecutive cycles (indicated with numbers) with no pause between the cycles. Blue circles indicate domain unfolding events. Inset is a magnified view of the initial part of the force trace to reveal step-wise transitions. (B) AFM images of two rabbit $m$. longissimus dorsi titin molecules stretched with receding meniscus. Scale bar: $200 \mathrm{~nm}$. P, the putative PEVK domain; M, M-line end of titin (Tskhovrebova and Trinick, 1997). Arrowheads indicate gaps in the topographical image corresponding to sites of domain unfolding. (C) Singlemolecule mechanics experiment on skeletal muscle titin in the absence (left, Control) and in the presence (right, PEVK fragment) of $24 \mu \mathrm{g} / \mathrm{ml}$ BAM3 PEVK domain fragment. Force curves were recorded on the same molecule prior to and following the addition of the PEVK fragment. 1st and 2nd stretches refer to subsequent mechanical cycles with no interleave pause. (D) Force response to ramp lengthening, with 2.5 fiber-length/second, imposed on a permeabilized rabbit $m$. psoas fiber in relaxing solution containing increasing concentrations of the polyE PEVK-domain fragment. Ramp lengthening was applied after 5 minutes of incubation. Temperature, $12.1^{\circ} \mathrm{C}$. Passive force traces are shown as a function of time. Inset shows force versus extension plot of the same dataset as in the main figure. Arrowheads indicate the onset of transition in the force trace.

$(n=150)$ could we observe regions that might be identified as the PEVK domain, but even these regions were much shorter than the contour length of the domain. Strikingly, however, all the 
molecules were scattered with topographical gaps along the entire contour, which probably correspond to regions in which one or two globular domains were unfolded. Although PEVK could be conformationally trapped (by surface interactions), globulardomain unfolding indeed precedes the complete extension of PEVK. There is a long-standing argument as to whether globulardomain unfolding or viscous PEVK extension is responsible for stress relaxation in muscle. Our findings thus suggest that both mechanisms contribute to the phenomenon. Finally, we explored whether structural transitions, such as the rupture of intra-PEVK interactions, might also take place in the cellular context, within the sarcomere. Force-driven intracellular protein unfolding has recently been detected by a shotgun labeling approach followed by mass-spectrometry analysis (Johnson et al., 2007). Here, we tested whether the low-force transitions, which are expected to contribute to a viscous or viscoelastic behavior at the level of the relaxed sarcomere, might be chased off with recombinant fragments of the PEVK domain (Fig. 7C,D; supplementary material Fig. S10). If certain regions of PEVK take part in binding reactions, the internal binding might be inhibited by the addition of soluble, small-molecule PEVK fragments that mimic the internal sequences. If the internal PEVK interactions cause an apparent chain shortening, then such a chase is expected to abolish chain shortening and hence reduce passive force at a given sarcomere length. Control experiments on single molecules of skeletal muscle titin (Fig. 7C) revealed that the addition of BAM3, a glutamate-rich PEVK fragment (Huber et al., 2012), resulted in three noticeable changes in the force spectra: (1) a reduction of transition forces in the investigated extension range; (2) a shift of the stretch-force traces towards increasing extensions; and (3) nearly complete disappearance of molecular fatigue. Stretching a relaxed skeletal muscle fiber in a ramp protocol within the physiological sarcomere-length range (2.5$2.8 \mu \mathrm{m})$ revealed a viscoelastic response similar to the averagedout titin force curve (Kellermayer et al., 1997), which could be partitioned into two phases: an initial phase characterized by rapid rise in force followed by one during which the force increase slowed down (Fig. 7D). The viscous component of this response was demonstrated by the relaxation of force following the ramp protocol. The viscoelastic response of the muscle fiber could be inhibited, in a concentration-dependent manner, by pretreating the fibers with the glutamate-rich polyE PEVK fragment, similar to the results on single titin molecules. Considering that the polyE motif might also interact with F-actin (Bianco et al., 2007; Nagy et al., 2004), further work will be necessary to confirm the mechanistic detail of the contribution of the PEVK and globular domains to sarcomere mechanics in the low-force regime. However, the muscle-fiber mechanics results, together with the observations of single-molecule mechanics corroborate the notion that non-canonical structural transitions of titin, such as the rupture of intra-PEVK domain interactions, play an important role in sarcomere mechanics.

The history-dependent mechanical behavior of titin might serve as a mechanosensor only if information is relayed towards signal-transduction pathways, which are typically cascades of molecular recognition and binding (Krüger and Linke, 2009; Linke, 2007; Ottenheijm et al., 2011; Voelkel and Linke, 2011). Conceivably, the low-force structural transitions in titin change the exposure of sites to phosphorylation and the binding of ligands. The mechanical opening of cryptic binding sites in multimodular proteins (Johnson et al., 2007) is thought to be an important mechanosensory mechanism (Vogel, 2006). Such a process might take place in titin. The self-zippering chain mechanism proposed here might serve as a universal mechanism for the history-dependent mechanical behavior in multimodular filamentous biomolecular systems.

In summary, the low-force structural transitions in titin observed in the present work might contribute to rapid, short-spatial-scale contourlength adjustments on the second time scale, hence within one contraction-relaxation cycle of the sarcomere. More importantly, the pattern of the transitions (number of steps, transition forces, contourlength increments) reflects the mechanical history of the sarcomere on the minute time scale. The mechanical history of the sarcomere probably depends on the long-timescale dynamics, such as the time spent by the sarcomere at a given length or contractile state. By reporting on the long-timescale sarcomeric status, the low-force structural transitions in titin could thus play an important role in the history-dependent rearrangements of sarcomeric structure, thereby contributing to a general mechanosensory function.

\section{MATERIALS AND METHODS}

\section{Preparation of proteins}

Skeletal muscle titin was prepared from rabbit $m$. longissimus dorsi by using previously published protocols (Kellermayer et al., 1997; Soteriou et al., 1993). Cardiac muscle titin was prepared from rabbit myocardium by steps identical to those of the skeletal muscle titin purification procedure but with buffer volumes scaled down according to initial tissue mass. Use of rabbit as the source of specimen was approved by the Regional Ethics Committee (approval number: XIV-I-001/29-7/2012). Purified titin samples were stored on ice in the presence of protease inhibitors (40 $\mu \mathrm{g} / \mathrm{ml}$ leupeptin, $20 \mu \mathrm{M}$ E64) until further use. The titin samples contained $>50 \%$ full-length, T1 titin (supplementary material Fig. S1). Typically, samples were used within 2 weeks of purification. The BAM3 PEVK fragment used in the single-molecule 'chase' experiments was prepared as described earlier (Huber et al., 2012). The recombinant $\mathrm{PEVK}$ fragment ('polyE'), used in muscle fiber mechanics experiments, was cloned from human titin cDNA and expressed in E.coli according to protocols established earlier (Nagy et al., 2005; Nagy et al., 2004). Except where noted otherwise, all chemicals were obtained from Sigma-Aldrich.

\section{Single-molecule manipulation}

For nanomanipulation, the Z-line end of titin was captured with a $3.0 \mu \mathrm{m}$ carboxylated latex bead (Kisker Biotech $\mathrm{GmbH}$, Steinfurt, Germany) coated with the T12 anti-titin antibody (Fürst et al., 1988). The other bead used was a $2.5 \mu \mathrm{m}$ amino-modified latex bead (Kisker Biotech $\mathrm{GmbH}$, Steinfurt, Germany) coated with the photoreactive crosslinker sulfoSANPAH (Thermo Scientific, Kvalitex, Hungary), providing a nonsequence-specific covalent linkage. One of the beads was captured in the optical trap, whereas the other was held with a micropipette embedded in a custom-built flow chamber mounted on a close-loop piezoelectric (PZT) stage (Nano-PDQ375, Mad City Labs, Madison, WI). Nanomechanical manipulation of titin was carried out with a custombuilt dual-beam counter-propagating optical tweezers apparatus (Kellermayer et al., 1997; Smith et al., 2003) in either constant velocity (velocity-clamp) or constant force (force-clamp) mode (Bianco et al., 2011). In constant-velocity mode, titin was stretched by moving the micropipette away from the trap with a constant rate (typically $250 \mathrm{~nm} /$ second) at a step-resolution of $\sim 1.1 \mathrm{~nm}$. In force-clamp mode the force was held near a set point by stretching or extending titin by rapid movement (typically $10 \mu \mathrm{m} / \mathrm{second}$ ) of the PZT stage with customwritten PID (proportional, integrating, differential) routines (bandwidth limited to $2.5 \mathrm{kHz}$ by the resonance frequency of the stage). Trap stiffness was $\sim 0.2 \mathrm{pN} / \mathrm{nm}$. Instrument control and data acquisition were managed by using custom-written LabView routines. Force was measured by calculating the change in photonic momentum (Smith et al., 2003) with a resolution of $\sim 0.5 \mathrm{pN}$. Buffer condition was $25 \mathrm{mM}$ imidazole- $\mathrm{HCl}$ ( $\mathrm{pH} 7.4), 200 \mathrm{mM} \mathrm{KCl}, 4 \mathrm{mM} \mathrm{MgCl} 2,1 \mathrm{mM}$ EGTA, 
$1 \mathrm{mM}$ DTT, $20 \mu \mathrm{g} / \mathrm{ml}$ leupeptin, $10 \mu \mathrm{M}$ E-64, $0.1 \% \mathrm{NaN}_{3}, 0.2 \%$ Tween20. The exchange of buffer solution (e.g. to lower ionic strength or to treat the molecule with 9D10 antibody, Developmental Studies Hybridoma Bank, University of Iowa) around a captured titin molecule was carried out with a custom-built, computer-controlled pressureregulation microfluidic system. Solution exchange was carried out with an already captured titin molecule present, to avoid deleterious titin aggregation and precipitation (Kellermayer et al., 2003).

\section{Atomic force microscopy}

Titin molecules stretched with receding meniscus (Tskhovrebova and Trinick, 1997; Tskhovrebova and Trinick, 2001) were imaged with a high-resolution atomic force microscope (Cypher, AsylumResearch, Santa Barbara, CA). Titin was diluted with PBS solution containing $50 \%$ glycerol to an approximate final protein concentration of $20 \mu \mathrm{g} / \mathrm{ml}$. $20 \mu \mathrm{l}$ sample was applied to freshly cleaved mica and immediately spun in a custom-built rotor at 13,000 r.p.m. for 10 seconds. The surface was extensively washed with distilled $\mathrm{H}_{2} \mathrm{O}$ and dried with clean $\mathrm{N}_{2}$ gas. Images were collected in tapping mode with a stiff cantilever (Olympus AC1560 or AC55TS) at a pixel resolution of $0.5-2 \mathrm{~nm}$.

\section{Single muscle fiber experiments}

Single permeabilized muscle fibers obtained from $m$. psoas of adult male New Zealand white rabbits were processed and manipulated according to protocols published earlier (Goldman et al., 1984; Linari et al., 2007; Piazzesi et al., 2007). Each end of a 5- to 6-mm-long segment of a single muscle fiber was attached using T-shaped aluminium clips to a linear, loudspeaker-based motor and a capacitance-based force transducer (resonance frequency $50 \mathrm{kHz}$ ), respectively (Lombardi and Piazzesi, 1990). The fiber was placed in relaxing solution (100 mM TES, pH 7.1, $7.7 \mathrm{mM} \mathrm{MgCl}_{2}, 5.44 \mathrm{mM}$ ATP, $25 \mathrm{mM}$ EGTA, $19.11 \mathrm{mM}$ creatine phosphate, $10 \mathrm{mM}$ glutathione). Sarcomere length $(s l)$, width $(w)$ and height $(h)$ of the fiber were measured at $0.5 \mathrm{~mm}$ intervals within the 3-4 mm central segment of the relaxed fiber by using a $40 \times$ objective lens (Zeiss, NA 0.60 ) and a $25 \times$ eyepiece. The fiber length $\left(L_{0}\right)$ was adjusted to a $s l$ of $2.5-2.8 \mu \mathrm{m}$. The fiber cross-sectional area (CSA) was determined by assuming an elliptical cross-section shape $(\mathrm{CSA}=\pi / 4 \cdot w \cdot h)$. Lateral filament spacing in the fiber was restored to the pre-permeabilization values by using the osmotic agent dextran T-500 (4 g/100 ml). A ramp lengthening (5\% amplitude and 2.5 fiber-length/second velocity) was applied to the relaxed fiber to elicit the passive force response. Mechanical stretch protocol was carried out in relaxing solution containing different concentrations of polyE fragment $(0,5,10,20 \mu \mathrm{g} / \mathrm{ml})$.

\section{Data processing and analysis}

In constant-velocity experiments, data were collected at acquisition rates of $1-5 \mathrm{kHz}$. In force-clamp experiments, the data acquisition rate was $0.5-1 \mathrm{kHz}$. To identify the zero-extension position in the displacement data, the micropipette bead was pressed gently against the trapped bead either before or following the nanomanipulation experiment (cf. Fig. 1A, inset). Molecular extension $(z)$ was calculated by correcting displacement $(s)$ according to force $(F)$ and trap stiffness $(\kappa)$ as

$$
z=s-\frac{F}{\kappa} .
$$

Data were usually smoothed to $100 \mathrm{~Hz}$ by using a median filter. Parts of the stretch curves were fitted with the worm like chain (WLC) equation

$$
\frac{F L_{P}}{k_{B} T}=\frac{z}{L}+\frac{1}{4\left(1-z / L_{C}\right)^{2}}-\frac{1}{4},
$$

where $L_{P}$ and $L_{C}$ are the persistence and the contour lengths, respectively, $k_{B}$ is Boltzmann's constant and $T$ is absolute temperature (Bustamante et al., 1994). Curve fitting was carried out by using the MarquardtLevenberg non-linear least squares method. Load-dependent transition rates (see Fig. 3) were fitted with the equation

$$
k_{F}=k_{0} e^{\frac{-F \Delta x}{k_{B} T}}
$$

where $k_{F}$ is the reaction rate in the presence of force, $k_{0}$ is the spontaneous, thermally activated reaction rate, and $\Delta x$ is the distance, along the reaction coordinate, between the stable, contracted state and the transition state (Bell, 1978; Evans and Ritchie, 1999). In the present work, analysis is presented based on data collected on 170 skeletal muscle and 15 cardiac titin molecules. For each molecule, 2-10 mechanical cycles were carried out; thus, the conclusions of this paper are based on about 1100 force curves. Data were processed with LabView (v.7.1), IgorPro (v.6.2.2.2) and KaleidaGraph (v.4.2) program packages.

\section{Modeling and simulation}

Globular domain and PEVK unfolding/refolding under force was modeled with Monte-Carlo simulations based on previously used simulation algorithms (Kellermayer et al., 1997; Kellermayer et al., 2000). The typical model molecule contained 50 globular domains and a 700-nm-long PEVK domain, the apparent contour length of which is shortened owing to the structural features of its $28 \mathrm{mer}$ units (Greaser, 2001; Kellermayer et al., 2003). As the chain was extended, force was generated according to the WLC equation (Bustamante et al., 1994). At each step, for the given force, the number of domains and repeat units unfolded/refolded were calculated according to (Bell, 1978; Evans and Ritchie, 1997):

$$
d N=N \omega_{0} d t e^{-\left(E_{a}-f \Delta x\right) / k_{B} T},
$$

where $d N$ is the number of 28-mer units or domains unfolded/refolded during the $d t$ polling interval, and $N$ is the number of available bonds or folded domains. In case of fractional $d N$, the process was permitted or prohibited depending on a comparison with a number generated randomly between 0 and 1. 28-mer and globular-domain unfolding or refolding events incremented or decremented the chain length with $11 \mathrm{~nm}$ and $28 \mathrm{~nm}$, respectively. $E_{a}$ and $\Delta x$ of globular-domain unfolding/refolding were $1.08 \times 10^{-19} / 8 \times 10^{-20} \mathrm{~J} /$ molecule and $0.3 / 8 \mathrm{~nm}$, respectively. $E_{a}$ of PEVK 28-mer unfolding/refolding were $1.05 \times 10^{-19} / 8 \times 10^{-20} \mathrm{~J} /$ molecule. $\Delta x$ of PEVK 28 -mer unfolding was randomly chosen between 0.3 and $0.5 \mathrm{~nm}$, and $\Delta x$ of refolding was set at $11 \mathrm{~nm}$.

\section{Acknowledgements}

The authors gratefully acknowledge the assistance of Ágnes Iszlai and Katalin Naftz with the preparation of titin and of István Derka with various aspects of designing the optical tweezers instrument. The human titin cDNA library was a generous gift from Siegfried Labeit (University of Heidelberg, Medical Faculty Mannheim). The T12 anti-titin antibody was a generous gift from Dieter Fürst (University of Bonn, Institute of Cell Biology). The insightful comments of Ricardo $H$. Pires and Levente Herényi on various stages of the manuscript preparation are gratefully acknowledged.

\section{Competing interests}

The authors declare no competing interests.

\section{Author contributions}

Z.M. designed experiments, conducted experiments, analyzed data and wrote the manuscript. P.B. designed and conducted experiments. M.L. designed and conducted muscle-fiber experiments. M.C. designed and conducted muscle-fiber experiments. A.N. cloned and expressed protein fragments. V.L. designed muscle-fiber experiments and analyzed data. M.K. designed experiments, analyzed data and wrote manuscript.

\section{Funding}

This work was supported by grants from the Hungarian Science Foundation [grant numbers OTKA K84133, OTKA K109480]. The research leading to these results has received funding from the European Union's Seventh Framework Program FP7/2007-2013 [grant number HEALTH-F2-2011-278850 (INMiND)].

\section{Supplementary material}

Supplementary material available online at

http://jcs.biologists.org/lookup/suppl/doi:10.1242/jcs.138461/-/DC1 


\section{References}

Anderson, B. R. and Granzier, H. L. (2012). Titin-based tension in the cardiac sarcomere: molecular origin and physiological adaptations. Prog. Biophys. Mol. Biol. 110, 204-217.

Bang, M. L., Centner, T., Fornoff, F., Geach, A. J., Gotthardt, M., McNabb, M., Witt, C. C., Labeit, D., Gregorio, C. C., Granzier, H. et al. (2001). The complete gene sequence of titin, expression of an unusual approximately 700 $\mathrm{kDa}$ titin isoform, and its interaction with obscurin identify a novel Z-line to I-band linking system. Circ. Res. 89, 1065-1072.

Bell, G. I. (1978). Models for the specific adhesion of cells to cells. Science $\mathbf{2 0 0}$ 618-627.

Bianco, P., Nagy, A., Kengyel, A., Szatmári, D., Mártonfalvi, Z., Huber, T. and Kellermayer, M. S. Z. (2007). Interaction forces between F-actin and titin PEVK domain measured with optical tweezers. Biophys. J. 93, 2102-2109.

Bianco, P., Bongini, L., Melli, L., Dolfi, M. and Lombardi, V. (2011). PicoNewtonmillisecond force steps reveal the transition kinetics and mechanism of the double-stranded DNA elongation. Biophys. J. 101, 866-874.

Bustamante, C., Marko, J. F., Siggia, E. D. and Smith, S. (1994). Entropic elasticity of lambda-phage DNA. Science 265, 1599-1600.

Bustamante, C., Macosko, J. C. and Wuite, G. J. (2000). Grabbing the cat by the tail: manipulating molecules one by one. Nat. Rev. Mol. Cell Biol. 1, 130-136.

Cecconi, C., Shank, E. A., Bustamante, C. and Marqusee, S. (2005). Direct observation of the three-state folding of a single protein molecule. Science 309, 2057-2060.

Elms, P. J., Chodera, J. D., Bustamante, C. and Marqusee, S. (2012). The molten globule state is unusually deformable under mechanical force. Proc. Natl. Acad. Sci. USA 109, 3796-3801.

Evans, E. (2001). Probing the relation between force-lifetime-and chemistry in single molecular bonds. Annu. Rev. Biophys. Biomol. Struct. 30, 105-128.

Evans, E. and Ritchie, K. (1997). Dynamic strength of molecular adhesion bonds Biophys. J. 72, 1541-1555.

Evans, E. and Ritchie, K. (1999). Strength of a weak bond connecting flexible polymer chains. Biophys. J. 76, 2439-2447.

Fisher, T. E., Carrion-Vazquez, M., Oberhauser, A. F., Li, H., Marszalek, P. E. and Fernandez, J. M. (2000). Single molecular force spectroscopy of modular proteins in the nervous system. Neuron 27, 435-446.

Freiburg, A., Trombitás, K., Hell, W., Cazorla, O., Fougerousse, F., Centner, T., Kolmerer, B., Witt, C., Beckmann, J. S., Gregorio, C. C. et al. (2000). Series of exon-skipping events in the elastic spring region of titin as the structural basis for myofibrillar elastic diversity. Circ. Res. 86, 1114-1121.

Funatsu, T., Higuchi, H. and Ishiwata, S. (1990). Elastic filaments in skeleta muscle revealed by selective removal of thin filaments with plasma gelsolin. J. Cell Biol. 110, 53-62.

Fürst, D. O., Osborn, M., Nave, R. and Weber, K. (1988). The organization of titin filaments in the half-sarcomere revealed by monoclonal antibodies in immunoelectron microscopy: a map of ten nonrepetitive epitopes starting at the $Z$ line extends close to the M line. J. Cell Biol. 106, 1563-1572.

Garcia-Manyes, S., Brujić, J., Badilla, C. L. and Fernández, J. M. (2007). Forceclamp spectroscopy of single-protein monomers reveals the individual unfolding and folding pathways of 127 and ubiquitin. Biophys. J. 93, 2436-2446.

Garcia-Manyes, S., Dougan, L., Badilla, C. L., Brujic, J. and Fernández, J. M. (2009). Direct observation of an ensemble of stable collapsed states in the mechanical folding of ubiquitin. Proc. Natl. Acad. Sci. USA 106, 10534-10539.

Gautel, M. (2010). The sarcomeric cytoskeleton: who picks up the strain? Curr. Opin. Cell Biol. 23, 39-46.

Goldman, Y. E., Hibberd, M. G. and Trentham, D. R. (1984). Relaxation of rabbit psoas muscle fibres from rigor by photochemical generation of adenosine-5' triphosphate. J. Physiol. 354, 577-604.

Grama, L., Nagy, A., Scholl, C., Huber, T. and Kellermayer, M. S. Z. (2005) Local variability in the mechanics of Titin's Tandem Ig segments. Croat. Chem. Acta 78, 405-411.

Granzier, H. L. and Labeit, S. (2005). Titin and its associated proteins: the third myofilament system of the sarcomere. Adv. Protein Chem. 71, 89-119.

Greaser, M. (2001). Identification of new repeating motifs in titin. Proteins 43, 145-149.

Gregorio, C. C., Granzier, H., Sorimachi, H. and Labeit, S. (1999). Muscle assembly: a titanic achievement? Curr. Opin. Cell Biol. 11, 18-25.

Helmes, M., Trombitás, K., Centner, T., Kellermayer, M., Labeit, S., Linke, W. A. and Granzier, H. (1999). Mechanically driven contour-length adjustment in rat cardiac titin's unique N2B sequence: titin is an adjustable spring. Circ. Res. 84, 1339-1352.

Herzog, J. A., Leonard, T. R., Jinha, A. and Herzog, W. (2012). Are titin properties reflected in single myofibrils? J. Biomech. 45, 1893-1899.

Higuchi, H., Nakauchi, Y., Maruyama, K. and Fujime, S. (1993). Characterization of beta-connectin (titin 2) from striated muscle by dynamic light scattering. Biophys. J. 65, 1906-1915.

Huber, T., Grama, L., Hetényi, C., Schay, G., Fülöp, L., Penke, B. and Kellermayer, M. S. Z. (2012). Conformational dynamics of titin PEVK explored with FRET spectroscopy. Biophys. J. 103, 1480-1489.

Improta, S., Politou, A. S. and Pastore, A. (1996). Immunoglobulin-like modules from titin I-band: extensible components of muscle elasticity. Structure 4, 323337.

Johnson, C. P., Tang, H. Y., Carag, C., Speicher, D. W. and Discher, D. E. (2007). Forced unfolding of proteins within cells. Science 317, 663-666.

Kellermayer, M. S., Smith, S. B., Granzier, H. L. and Bustamante, C. (1997) Folding-unfolding transitions in single titin molecules characterized with laser tweezers. Science 276, 1112-1116.
Kellermayer, M. S., Smith, S. B., Bustamante, C. and Granzier, H. L. (1998). Complete unfolding of the titin molecule under external force. J. Struct. Biol. 122, 197-205.

Kellermayer, M. S., Smith, S., Bustamante, C. and Granzier, H. L. (2000). Mechanical manipulation of single titin molecules with laser tweezers. Adv. Exp. Med. Biol. 481, 111-126, discussion 127-128.

Kellermayer, M. S., Smith, S. B., Bustamante, C. and Granzier, H. L. (2001). Mechanical fatigue in repetitively stretched single molecules of titin. Biophys. J. 80, 852-863

Kellermayer, M. S. Z., Bustamante, C. and Granzier, H. L. (2003). Mechanics and structure of titin oligomers explored with atomic force microscopy. Biochim. Biophys. Acta 1604, 105-114.

Krüger, M. and Linke, W. A. (2009). Titin-based mechanical signalling in normal and failing myocardium. J. Mol. Cell. Cardiol. 46, 490-498.

Labeit, S. and Kolmerer, B. (1995). Titins: giant proteins in charge of muscle ultrastructure and elasticity. Science 270, 293-296.

Leake, M. C., Wilson, D., Gautel, M. and Simmons, R. M. (2004). The elasticity of single titin molecules using a two-bead optical tweezers assay. Biophys. J. 87, 1112-1135

Li, H., Oberhauser, A. F., Redick, S. D., Carrion-Vazquez, M., Erickson, H. P. and Fernandez, J. M. (2001). Multiple conformations of PEVK proteins detected by single-molecule techniques. Proc. Natl. Acad. Sci. USA 98, 1068210686.

Li, H., Linke, W. A., Oberhauser, A. F., Carrion-Vazquez, M., Kerkvliet, J. G., Lu, H., Marszalek, P. E. and Fernandez, J. M. (2002). Reverse engineering of the giant muscle protein titin. Nature 418, 998-1002.

Linari, M., Caremani, M., Piperio, C., Brandt, P. and Lombardi, V. (2007). Stiffness and fraction of Myosin motors responsible for active force in permeabilized muscle fibers from rabbit psoas. Biophys. J. 92, 2476-2490.

Linke, W. A. (2000). Titin elasticity in the context of the sarcomere: force and extensibility measurements on single myofibrils. Adv. Exp. Med. Biol. 481, 179202, discussion 203-206.

Linke, W. (2007). Sense and stretchability: The role of titin and titin-associated proteins in myocardial stress-sensing and mechanical dysfunction. Cardiovasc. Res. 77, 637-648.

Linke, W. A. and Leake, M. C. (2004). Multiple sources of passive stress relaxation in muscle fibres. Phys. Med. Biol. 49, 3613-3627.

Linke, W. A., Ivemeyer, M., Olivieri, N., Kolmerer, B., Rüegg, J. C. and Labeit, S. (1996). Towards a molecular understanding of the elasticity of titin. J. Mol. Biol. 261, 62-71.

Linke, W. A., Ivemeyer, M., Mundel, P., Stockmeier, M. R. and Kolmerer, B. (1998a). Nature of PEVK-titin elasticity in skeletal muscle. Proc. Natl. Acad. Sci. USA 95, 8052-8057.

Linke, W. A., Stockmeier, M. R., Ivemeyer, M., Hosser, H. and Mundel, P. (1998b). Characterizing titin's I-band Ig domain region as an entropic spring. J. Cell Sci. 111, 1567-1574.

Linke, W. A., Rudy, D. E., Centner, T., Gautel, M., Witt, C., Labeit, S. and Gregorio, C. C. (1999). I-band titin in cardiac muscle is a three-element molecular spring and is critical for maintaining thin filament structure. J. Cell Biol. 146, 631-644.

Liversage, A. D., Holmes, D., Knight, P. J., Tskhovrebova, L. and Trinick, J. (2001). Titin and the sarcomere symmetry paradox. J. Mol. Biol. 305, 401409.

Lombardi, V. and Piazzesi, G. (1990). The contractile response during steady lengthening of stimulated frog muscle fibres. J. Physiol. 431, 141-171.

Ma, K. and Wang, K. (2003). Malleable conformation of the elastic PEVK segment of titin: non-co-operative interconversion of polyproline II helix, beta-turn and unordered structures. Biochem. J. 374, 687-695.

Maruyama, K. (1997). Connectin/titin, giant elastic protein of muscle. FASEB J. 11, 341-345.

Mártonfalvi, Z. and Kellermayer, M. S. (2014). Individual globular domains and domain unfolding visualized in overstretched titin molecules with atomic force microscopy. PLoS One. In press.

Minajeva, A., Kulke, M., Fernandez, J. M. and Linke, W. A. (2001). Unfolding of titin domains explains the viscoelastic behavior of skeletal myofibrils. Biophys. J. 80, 1442-1451.

Nagy, A., Cacciafesta, P., Grama, L., Kengyel, A., Málnási-Csizmadia, A. and Kellermayer, M. S. Z. (2004). Differential actin binding along the PEVK domain of skeletal muscle titin. J. Cell Sci. 117, 5781-5789.

Nagy, A., Grama, L., Huber, T., Bianco, P., Trombitás, K., Granzier, H. L. and Kellermayer, M. S. Z. (2005). Hierarchical extensibility in the PEVK domain of skeletal-muscle titin. Biophys. J. 89, 329-336.

Neuman, K. C. and Nagy, A. (2008). Single-molecule force spectroscopy: optical tweezers, magnetic tweezers and atomic force microscopy. Nat. Methods 5, 491-505.

Ottenheijm, C. A. C., van Hees, H. W. H., Heunks, L. M. A. and Granzier, H. (2011). Titin-based mechanosensing and signaling: role in diaphragm atrophy during unloading? Am. J. Physiol. Lung Cell Mol. Physiol. 300, L161-L166.

Piazzesi, G., Reconditi, M., Linari, M., Lucii, L., Bianco, P., Brunello, E., Decostre, V., Stewart, A., Gore, D. B., Irving, T. C. et al. (2007). Skeletal muscle performance determined by modulation of number of myosin motors rather than motor force or stroke size. Cell 131, 784-795.

Rief, M. and Grubmüller, H. (2002). Force spectroscopy of single biomolecules. Chemphyschem 3, 255-261. 
Rief, M., Gautel, M., Oesterhelt, F., Fernandez, J. M. and Gaub, H. E. (1997) Reversible unfolding of individual titin immunoglobulin domains by AFM Science 276, 1109-1112.

Rief, M., Fernandez, J. and Gaub, H. (1998a). Elastically coupled two-leve systems as a model for biopolymer extensibility. Phys. Rev. Lett. 81, 4764-4767.

Rief, M., Gautel, M., Schemmel, A. and Gaub, H. E. (1998b). The mechanical stability of immunoglobulin and fibronectin III domains in the muscle protein titin measured by atomic force microscopy. Biophys. J. 75, 3008-3014.

Smith, S. B., Cui, Y. and Bustamante, C. (2003). Optical-trap force transducer that operates by direct measurement of light momentum. Methods Enzymol. 361, 134-162.

Soteriou, A., Gamage, M. and Trinick, J. (1993). A survey of interactions made by the giant protein titin. J. Cell Sci. 104, 119-123.

Trombitás, K., Greaser, M., French, G. and Granzier, H. (1998a). PEVK extension of human soleus muscle titin revealed by immunolabeling with the anti-titin antibody 9D10. J. Struct. Biol. 122, 188-196.

Trombitás, K., Greaser, M., Labeit, S., Jin, J. P., Kellermayer, M., Helmes, M. and Granzier, H. (1998b). Titin extensibility in situ: entropic elasticity of permanently folded and permanently unfolded molecular segments. J. Cell Biol. 140, 853-859.

Trombitás, K., Redkar, A., Centner, T., Wu, Y., Labeit, S. and Granzier, H. (2000). Extensibility of isoforms of cardiac titin: variation in contour length of molecular subsegments provides a basis for cellular passive stiffness diversity. Biophys. J. 79, 3226-3234.

Trombitás, K., Wu, Y., McNabb, M., Greaser, M., Kellermayer, M. S., Labeit, S. and Granzier, H. (2003). Molecular basis of passive stress relaxation in human soleus fibers: assessment of the role of immunoglobulin-like domain unfolding. Biophys. J. 85, 3142-3153.

Tskhovrebova, L. and Trinick, J. (1997). Direct visualization of extensibility in isolated titin molecules. J. Mol. Biol. 265, 100-106.

Tskhovrebova, L. and Trinick, J. (2001). Flexibility and extensibility in the titin molecule: analysis of electron microscope data. J. Mol. Biol. 310, 755-771.

Tskhovrebova, L., Trinick, J., Sleep, J. A. and Simmons, R. M. (1997). Elasticity and unfolding of single molecules of the giant muscle protein titin. Nature 387, 308-312.

Voelkel, T. and Linke, W. A. (2011). Conformation-regulated mechanosensory control via titin domains in cardiac muscle. Pflugers Arch. 462, 143-154.

Vogel, V. (2006). Mechanotransduction involving multimodular proteins: converting force into biochemical signals. Annu. Rev. Biophys. Biomol. Struct. 35, 459-488.

Wang, K. (1996). Titin/connectin and nebulin: giant protein rulers of muscle structure and function. Adv. Biophys. 33, 123-134.

Wells, R. G. (2013). Tissue mechanics and fibrosis. Biochim. Biophys. Acta 1832 , 884-890. 